\title{
PROPOSTA DE INDICADORES DE RESPOSTAS ESTRATÉGICAS ÀS PRESSÕES INSTITUCIONAIS PELA SUSTENTABILIDADE EM EMPRESAS INTERNACIONALIZADAS
}

\author{
PROPOSITION OF INDICATORS OF STRATEGIC RESPONSES TO \\ INSTITUTIONAL PRESSURES FOR SUSTAINABILITY IN \\ INTERNATIONALIZED COMPANIES
}

\section{PROPUESTA DE INDICADORES DE RESPUESTAS ESTRATÉGICAS A PRESIONES INSTITUCIONALES PARA LA SOSTENIBILIDAD EN EMPRESAS INTERNACIONALIZADAS.}

Maríndia Brachak dos Santos

Doutora em Administração pelo Programa de Pós Graduação em Administração da Universidade Federal de Santa Maria marindiabrachak@gmail.com https://orcid.org/0000-0002-4078-9005

Flavia Luciane Scherer

Doutora em administração pela Universidade Federal de Minas Gerais (UFMG). Professora associada da Universidade Federal de Santa Maria e coordenadora do Curso de Administração.

flaviascherer@globo.com https://orcid.org/0000-0003-1581-2653

Nathália Rigui Trindade

Doutoranda em Administração no Programa de Pós Graduação em Administração da Universidade Federal de Santa Maria nathaliariguitrindade@gmail.com https://orcid.org/0000-0003-1361-6501

Maíra Nunes Piveta

Doutoranda em Administração no Programa de Pós Graduação em Administração da Universidade Federal de Santa Maria mairanpiveta@gmail.com https://orcid.org/0000-0001-5518-9515

$$
\begin{aligned}
& \text { Editor Científico: José Edson Lara } \\
& \text { Organização Comitê Científico } \\
& \text { Double Blind Review pelo SEER/OJS } \\
& \text { Recebido em 15.05.2020 } \\
& \text { Aprovado em 18.10.2020 }
\end{aligned}
$$




\begin{abstract}
Objective of the study: To build indicators for measuring strategic responses to institutional pressures for sustainability in internationalized companies in the furniture sector.

Methodology/approach: The study was carried out in two stages. The first stage consisted of an exploratory research with a qualitative approach through interviews with three companies in the furniture sector in Rio Grande do Sul. The quantitative stage was accomplished through the survey method with the instrument previously developed. The data were analyzed using exploratory factor analysis.
\end{abstract}

Originality/Relevance: When considering the institutional perspective and the emergence and transversality of the themes of sustainability and internationalization, the study explores a theoretical gap that has aroused the interest of researchers from different fields of knowledge.

Main results: The results showed that the model tested was statistically adequate for measuring the five dimensions, originally proposed by Oliver (1991). The five dimensions mentioned above are operationalized in 15 observable variables, capable of measuring strategic responses to institutional pressures for sustainability, which explain $67.89 \%$ of the total data variance.

Theoretical / methodological contributions: Contributes to the proposal and application of a quantitative research instrument for strategic responses to institutional pressures for sustainability. According to the literature on the subject, studies that approach the perspective of strategic responses, proposed by Oliver (1991), are mostly qualitative studies. Another important contribution is the attempt to relate constructs in a way not yet addressed in the literature on internationalization, highlighting two important themes (degree of internationalization and sustainability).

Keywords: Strategic responses. Institutional pressures. Sustainability. Internationalization. Furniture sector.

\title{
Resumo
}

Objetivo do estudo: Desenvolver indicadores para medir as respostas estratégicas às pressões institucionais pela sustentabilidade em empresas internacionalizadas do setor moveleiro.

Metodologia / abordagem: o estudo foi realizado em duas etapas. A primeira etapa consistiu em uma pesquisa exploratória com abordagem qualitativa por meio de entrevistas com três empresas do setor moveleiro do Rio Grande do Sul. A etapa quantitativa foi realizada por meio do método survey com o instrumento previamente desenvolvido. Os dados foram analisados por meio de análise fatorial exploratória.

Originalidade / Relevância: Considerando a perspectiva institucional e a emergência e transversalidade das questões de sustentabilidade e internacionalização, o estudo explora uma lacuna teórica que tem despertado o interesse de pesquisadores de diferentes áreas do conhecimento. 
Principais resultados: Os resultados mostraram que o modelo testado foi estatisticamente adequado para medir as cinco dimensões, propostas originalmente por Oliver (1991). As cinco dimensões mencionadas acima são operacionais em 15 variáveis observáveis, capazes de medir as respostas estratégicas às pressões institucionais por sustentabilidade, o que explica $67,89 \%$ da variância total dos dados.

Contribuições teórico-metodológicas: Contribui para a proposição e aplicação de um instrumento de pesquisa quantitativa para respostas estratégicas às pressões institucionais pela sustentabilidade. De acordo com a literatura sobre o assunto, os estudos que abordam a perspectiva de respostas estratégicas, proposta por Oliver (1991), são em sua maioria estudos qualitativos. Outra contribuição importante é a tentativa de relacionar as construções de uma forma ainda não abordada na literatura sobre internacionalização, destacando duas questões importantes (grau de internacionalização e sustentabilidade).

Palavras-chave: Respostas estratégicas. Pressões institucionais. Sustentabilidade Internacionalização do setor moveleiro.

\section{Resumen}

Objetivo del estudio: Desarrollar indicadores para medir las respuestas estratégicas a las presiones institucionales para la sostenibilidad en empresas internacionalizadas en el sector del mueble.

Metodología/enfoque: el estudio se realizó en dos etapas. La primera etapa consistió en una investigación exploratoria con un enfoque cualitativo a través de entrevistas con tres empresas del sector del mueble en Rio Grande do Sul. La etapa cuantitativa se realizó a través del método de encuesta con el instrumento previamente desarrollado. Los datos se analizaron mediante análisis factorial exploratorio.

Originalidad/Relevancia: Al considerar la perspectiva institucional y el surgimiento y transversalidad de los temas de sostenibilidad e internacionalización, el estudio explora una brecha teórica que ha despertado el interés de investigadores de diferentes campos del conocimiento.

Resultados principales: Los resultados mostraron que el modelo probado fue estadísticamente adecuado para medir las cinco dimensiones, originalmente propuesto por Oliver (1991). Las cinco dimensiones mencionadas anteriormente están operativas en 15 variables observables, capaces de medir las respuestas estratégicas a las presiones institucionales para la sostenibilidad, lo que explica el $67.89 \%$ de la varianza total de datos.

Contribuciones teóricas/metodológicas: Contribuye a la propuesta y aplicación de un instrumento de investigación cuantitativa para respuestas estratégicas a presiones institucionales para la sostenibilidad. Según la literatura sobre el tema, los estudios que abordan la perspectiva de las respuestas estratégicas, propuestas por Oliver (1991), son en su mayoría estudios cualitativos. Otra contribución importante es el intento de relacionar construcciones de una manera que aún no se aborda en la literatura sobre internacionalización, destacando dos temas importantes (grado de internacionalización y sostenibilidad). 
Palabras clave: Respuestas estratégicas. Presiones institucionales. Sustentabilidad. Internacionalización Sector del mueble.

\section{INTRODUÇÃO}

Diante do atual panorama global de permanentes transformações, no qual questões como desigualdades sociais, degradação ambiental, e globalização têm influenciado direta e indiretamente a maior parte dos setores da atividade humana, emerge a necessidade de repensar hábitos, valores e processos. Esta realidade tem evidenciado a importância que a sustentabilidade tem desempenhado na gestão de empresas multinacionais de mercados emergentes (Ciasullo, Montera, Cucari, \& Polese, 2020). Dadas as circunstâncias globais, conhecer o ambiente organizacional e o processo de tomada de decisão frente a alguns impasses, representa uma nova perspectiva de estudos institucionais. As decisões organizacionais perpassam entre ceder ou oferecer resistência às pressões institucionais, sejam elas recebidas do mercado, governo, sociedade e de seu próprio segmento para a institucionalização de práticas sustentáveis (Carvalho, 2010).

Observa-se, assim, que o impacto das instituições nas organizações tem conferido à Teoria Institucional o ponto de partida para numerosos estudos que sugerem que as decisões organizacionais são moldadas de significados sociais e valores socialmente construídos, cujo objetivo central é obter legitimidade ou conformidade às normas e aos padrões institucionais estabelecidos (Deng \& Zhang, 2018; Luo \& Zhang, 2016; Ahmed \& Brennan, 2019).

As normas e padrões institucionais fazem com que as organizações passem a condicionar, ou mesmo determinar, as suas escolhas estratégicas em virtude das pressões sofridas no ambiente institucional (DiMaggio \& Powell, 2005). Com a finalidade de entender as escolhas estratégicas realizadas pelas organizações, Oliver (1991) propôs uma tipologia de respostas estratégicas ao processo institucional e sugere que estas irão variar de conformidade à resistência, de passiva à ativa, de pré-consciente à controlada, de impotente à influente e de habitual à oportunista, dependendo das pressões por conformidade que são exercidas sobre as organizações. Nota-se que a tipologia proposta por Oliver (1991) continua em voga em muitos estudos, muito embora, o seu emprego tenha privilegiado uma perspectiva mais qualitativa, como os trabalhos desenvolvidos por Scherer (2007) e Machado-da-Silva e Fonseca (2010) no Brasil, além da pesquisa de Sutter, Webb, Kistruck \& Bailey (2013) na Guatemala. 
Com base nas respostas estratégicas instituídas, verifica-se que as organizações são desafiadas a posicionar-se em vários contextos, dependendo dos locais e das relações que a empresa desenvolve. Observa-se, assim, que são muitos os atores sociais envolvidos, o que dificulta as respostas organizacionais, sendo que estas interações acontecem tanto em nível nacional como internacional. A este respeito, Fleury e Fleury (2012) indicam que o ambiente internacional é ainda mais complexo, pois os atores envolvidos contribuem para as diferenciações e similaridades do mercado.

Dado o exposto, inserir-se no mercado internacional requer o enfrentamento de diversos desafios, visto que as cobranças para adequação às normas e regras institucionalizadas por agentes econômicos, sociais e ambientais têm gerado dificuldades para muitas empresas brasileiras (Carvalho, Vieira \& Silva, 2012). Ciasullo et al. (2020) destacam, no entanto, que o arcabouço teórico e empírico que abordam a sustentabilidade e a internacionalização de empresas ainda não tem sido amplamente explorado.

É notória a crescente pressão sobre as organizações, não somente em termos de legislação, mas também da sociedade que está cada vez mais atenta aos aspectos relacionados à sustentabilidade passando, assim, a avaliar o comportamento organizacional apoiadas pelo preceito do desenvolvimento sustentável. Atrelado a esses entraves tem-se, ainda, o desafio de buscar novos mercados por meio da internacionalização das atividades para além das fronteiras nacionais (Carneiro \& Dib, 2007; Fleury \& Fleury, 2012). Com relação a setores econômicos que têm sofrido pressões para adoção de práticas de sustentabilidade, destaca-se o moveleiro. Observa-se que o setor demonstra preocupação em virtude das crescentes restrições de caráter ambiental ao uso de madeiras de lei disseminadas internacionalmente (Aprendendo a Exportar, 2014). Outras normas e regras impostas ao setor para a internacionalização, como a adoção de gestão ambiental, barreiras econômicas e as dificuldades para a exportação, têm impactado na gestão das organizações e consequentemente no desenvolvimento de um grau mais avançado de internacionalização das empresas desse setor (Floriani, 2010).

Posto isto, o presente estudo dedica-se a atender o seguinte objetivo de pesquisa: construir indicadores para a mensuração das respostas estratégicas às pressões Institucionais para a sustentabilidade em empresas internacionalizadas do setor moveleiro. Para uma melhor organização, este estudo encontra-se dividido em sete seções sendo, além desta introdução, um apanhado sobre Teoria Institucional e Respostas Estratégicas, seguido da revisão da 
literatura sobre Sustentabilidade e Organizações bem como sobre a Internacionalização do Setor Moveleiro. Logo após, apresenta-se o método do estudo, a apresentação e discussão dos resultados e, por fim, as considerações finais. Finalmente, as referências utilizadas ao longo do estudo são apresentadas.

\section{TEORIA INSTITUCIONAL E RESPOSTAS ESTRATÉGICAS}

A visão da Teoria Institucional de acordo com Selznick (1972, p.5) pode ser definida como o "produto natural das pressões e necessidades sociais, um organismo adaptável e receptivo". Desta forma, assume-se que as organizações se tornam instituições quando assumem caráter especial e desenvolvem competências singulares em suas atividades. As instituições, por sua vez, constituem-se em estruturas e padrões de comportamento, formadas por elementos regulativos, normativos e culturais-cognitivos, que fornecem estabilidade e significado para o comportamento social, mas estão sujeitas a processos de mudanças incrementais e descontínuas (Scott, 2008). Diferencia-se, portanto de organizações, que são esquematizadas com o propósito de atingir uma lógica de eficiência, mobilizando as atividades por meio de instrumentos técnicos em prol de um conjunto de objetivos (Selznick, 1972).

Neste enfoque, em uma apreciação da Teoria Institucional Oliver (1991) enfatiza que diversos tipos de comportamentos estratégicos podem ser estabelecidos pelas organizações em resposta ao ambiente institucional. A autora sugere que as respostas organizacionais irão variar de conformidade à resistência, de passiva para ativa, dependendo das pressões por conformidade que são exercidas sobre as organizações. A partir de tais variações, Oliver (1991) apresentou uma tipologia de respostas estratégicas aos processos institucionais que estão divididas em cinco categorias, sendo elas aquiescência, compromisso, fuga, desafio e manipulação. A cada resposta estratégica, foram associadas três alternativas ou táticas, como se pode visualizar no Tabela 1. 


\section{Tabela 1}

Respostas estratégicas aos processos institucionais

\begin{tabular}{l|l|l}
\hline \multicolumn{1}{c|}{ Respostas Estratégicas } & \multicolumn{1}{c}{ Táticas } & \multicolumn{1}{c}{ Exemplos } \\
\hline Aquiescência & $\begin{array}{l}\text { Hábito } \\
\text { Imitação } \\
\text { Obediência }\end{array}$ & $\begin{array}{l}\text { Seguir normas invisíveis e tidas como verdadeiras. } \\
\text { Imitar modelos institucionais. } \\
\text { Obedecer a regras e aceitar as normas. }\end{array}$ \\
\hline Compromisso & $\begin{array}{l}\text { Equilíbrio } \\
\text { Pacificação } \\
\text { Barganha }\end{array}$ & $\begin{array}{l}\text { Equilibrar as expectativas de múltiplos atores. } \\
\text { Apaziguar e acomodar elementos institucionais. } \\
\text { Negociar com stakeholders institucionais. }\end{array}$ \\
\hline \multirow{2}{*}{ Fuga } & $\begin{array}{l}\text { Ocultação } \\
\text { Proteção } \\
\text { Escape }\end{array}$ & $\begin{array}{l}\text { Disfarçar a não conformidade. } \\
\text { Livrar-se de ligações institucionais. } \\
\text { Mudar objetivos, atividades ou domínios. }\end{array}$ \\
\hline \multirow{2}{*}{ Desafio } & $\begin{array}{l}\text { Liberação } \\
\text { Recusa } \\
\text { Ataque }\end{array}$ & $\begin{array}{l}\text { Ignorar normas e valores explícitos. } \\
\text { Contestar regras e requerimentos. } \\
\text { Atacar origens de pressões institucionais. }\end{array}$ \\
\hline Manipulação & $\begin{array}{l}\text { Cooptação } \\
\text { Influência } \\
\text { Controle }\end{array}$ & $\begin{array}{l}\text { Importar atores influentes. } \\
\text { Dar forma a valores e critérios. } \\
\text { Dominar atores e processos institucionais. }\end{array}$ \\
\hline
\end{tabular}

Fonte: Oliver (1991, p. 152)

A aquiescência representa o tipo de resposta estratégica mais passiva e ocorre sob as formas de hábito, imitação e obediência. A segunda estratégia de compromisso refere-se às demandas institucionais conflitantes e às inconsistências entre expectativas institucionais e objetivos organizacionais como os elementos motivadores da resposta estratégica. A terceira estratégia de respostas aos processos institucionais é a fuga, definida por Oliver (1991) como a tentativa da organização para evitar a necessidade de conformidade. A quarta é o desafio, que corresponde a uma forma mais ativa de resistência a processos institucionais. A quinta e última estratégia é a manipulação, a qual é a mais ativa resposta às pressões institucionais, em que se busca alterar ou exercer poder sobre o conteúdo das próprias expectativas ou fontes que buscam expressar ou aplicar a pressão (Oliver, 1991). Ressalta-se que estas categorias, segundo Machado-da-Silva, Fonseca, e Crubelatte (2010), não são mutuamente excludentes, isto é, a mesma empresa pode apresentar mais de uma única resposta à determinada pressão. Desta forma, observa-se que a tipologia proposta por Oliver (1991) amplia o entendimento das formas pelas quais as organizações respondem às pressões institucionais (Scherer, 2007). Defende-se assim, a premissa de que a resposta varia de um extremo de total passividade e submissão a um comportamento mais ativo de rejeição e tentativa de controle das forças institucionais.

A partir do exposto, salienta-se que apesar de ter sido elaborada há mais de duas décadas, a tipologia de Oliver (1991) ainda permanece atual. Estudos recentes como o trabalho de Cao e Pederzoli (2013) utilizam-se da ideia apresentada por Oliver para analisar $@ \oplus$ Revista Gestão \& Tecnologia, Pedro Leopoldo, v. 20, n.4, p. 202-226, out./dez.2020 
as respostas estratégicas dos varejistas internacionais para o ambiente institucional em mercados emergentes. Ao passo que, o estudo de Sutter et al., (2013) ponderaram como empresários da Guatemala respondem a arranjos institucionais envolvendo atores ilegítimos. O trabalho desenvolvido por (2013) também fez uso da tipologia de Oliver para estudar a ligação entre a instituição e estratégia, com o enfoque de pesquisar a relação existente entre a instituição proeminente na indústria do biodiesel e as estratégias de aquisição de matériaprima dos produtores de biodiesel da Região Sul do Brasil.

A próxima seção abordará o aporte teórico no que se refere a temática da sustentabilidade dentro das organizações.

\section{SUSTENTABILIDADE E ORGANIZAÇÕES}

As transformações ocorridas na sociedade e nos modos de produção, a partir da ideia de que os recursos naturais são finitos, tem direcionado as organizações a buscar novas formas de gerir seus negócios, passando a incluir a sustentabilidade na sua estratégia corporativa. Entretanto, inserir a sustentabilidade na estratégia da organização segundo Cunha, Spers, e Zylbersztajn (2011) é uma tarefa complexa, pois abrange um conjunto de fatores sociais, econômicos e ambientais que devem estar institucionalizados para permitir a qualificação da organização como sustentável.

Para tanto, as organizações sustentáveis podem ser consideradas aquelas que baseiam suas escolhas estratégicas corporativas que guiam suas ações em prol de inovações que promovam desenvolvimento econômico viável, de maneira a não agredir o meio ambiente e contribuir o desenvolvimento social da região e do país onde atuam. Com vistas a atender as transformações, as organizações passam a se concentrar no desenvolvimento de estratégia e práticas que incluam os três pilares da sustentabilidade social, ambiental e econômica na tomada de decisão organizacional (Ciasullo et al., 2020).

A este respeito, Barbieri, Vasconcelos, Andreassi \& Vasconcelos (2010) salientam que em princípio as organizações passaram a se inserir no movimento pelo desenvolvimento sustentável mais em razão das pressões externas, e como resposta às cobranças das entidades governamentais e da sociedade civil como um todo. No entanto, nas últimas décadas esta inserção tem sido vista como fator de competitividade empresarial podendo ser aporte de diferenciação ou qualificação no mercado e até significar a sobrevivência do negócio. 
Nesta perspectiva sustentável, John Elkington, em 1998, abordou o conceito do Triple Botton Line ou tripé da sustentabilidade em seu livro "Canibais com Garfo e Faca" propiciando uma reflexão sobre a gestão ligada à noção de desenvolvimento sustentável. Segundo o autor (1998, p. 397) “o desenvolvimento sustentável envolve a realização simultânea de prosperidade econômica, qualidade ambiental e equidade social". Desta forma, ressalta-se que, caso um dos princípios não seja alcançado, o desenvolvimento econômico não será sustentável.

Barbieri et al. (2010) observam ainda, que a explicação para a difusão do conceito de desenvolvimento sustentável no meio empresarial pode ser encontrada na Teoria Institucional a partir do argumento de Meyer e Rowan (1977) de que as organizações incorporam práticas institucionalizadas na sociedade, que se tornam mitos a serem seguidos, e visam aumentar a legitimidade e as condições de sobrevivência das organizações.

A partir do exposto, destaca-se que este estudo se utiliza da sustentabilidade sob a perspectiva do Triple Botton Line, compreendida pelas dimensões ambiental, social e econômica. Por ser a sustentabilidade dos recursos e a sua correta utilização uma preocupação global, a próxima seção dissertará sobre a internacionalização do setor moveleiro, visto que o estudo investigou empresas internacionalizadas do setor moveleiro.

\section{INTERNACIONALIZAÇÃO DO SETOR MOVELEIRO}

O novo cenário competitivo, a inclusão das economias emergentes, as crises nos países desenvolvidos, os avanços da tecnologia da informação e as mudanças no comportamento da sociedade têm levado as empresas a uma maior competitividade no mundo empresarial (Vidal, Barbosa, \& Bouzada, 2013). Contudo, mesmo diante destes novos desafios, as organizações visualizam a internacionalização como uma alternativa promissora para os seus negócios. Neste panorama, destaca-se a indústria de móveis no Brasil que avançou muito nos últimos anos, sendo que a sua produtividade, em alguns segmentos, já se aproxima dos níveis internacionais, resultado da abertura comercial e da globalização das atividades econômicas (Arruda, 2009). De acordo com o Banco Nacional de Desenvolvimento Econômico e Social (BNDES, 2013) inovações em logística e a abertura das economias modernas ao comércio internacional e aos investimentos produtivos contribuíram com o crescimento da internacionalização desta indústria. 
A expansão do setor moveleiro aliado à abertura de mercado possibilitou ao setor ampliar a visão mundial, fazendo com que o setor tivesse acesso e passasse a utilizar conceitos e tecnologias internacionais, que contribuíram com a modernização dos parques fabris do país, além de incentivar a evolução dos próprios fornecedores brasileiros (Maffessoni, 2012).

Observa-se assim, que a indústria de móveis tradicionalmente possui características comuns aos níveis internacionais, entre elas pode-se citar a utilização intensiva de mão de obra em relação a outros segmentos industriais, predomínio de pequenas e médias empresas, pequena participação no valor adicionado pela indústria de transformação, e as modificações no seu processo produtivo podem ser feitas de maneira incremental e por etapas, devido ao variado número de operações realizadas e produtos elaborados (Leão \& Naveiro, 2010).

Com relação às questões sustentáveis, observa-se que o setor moveleiro demonstra preocupação, em virtude das crescentes restrições de caráter ambiental ao uso de madeiras de lei (Aprendendo a Exportar, 2014). Essas restrições levam os fabricantes a buscarem e a utilizarem madeiras de reflorestamento no processo produtivo, em substituição a utilização de madeira maciça. Este fator torna-se um elemento de competitividade favorável à indústria brasileira em função da grande extensão de florestas, visto que o futuro da indústria de móveis reside no uso crescente desse tipo de matéria-prima. Esse diferencial competitivo, segundo a Abimóvel (2014), tem levado o governo brasileiro a discutir políticas de modernização para o setor, com vistas à sua inserção no mercado internacional.

Nesta direção, verifica-se que as exigências de certificações de caráter ambiental e a própria norma ISO 14000 deverão dificultar o mercado de móveis produzidos a partir de madeira de lei e estimular o uso de madeira de reflorestamento que se configura como uma tendência que ganha força no mercado mundial e, para a qual a indústria brasileira revela condições de competitividade (Aprendendo a Exportar, 2014). Ademais, o setor moveleiro brasileiro consiste em um dos pioneiros na obtenção do selo verde, o qual certifica que o móvel foi fabricado com matéria-prima oriunda de floresta de manejo sustentável.

Com base nas informações apresentadas, pondera-se que o setor moveleiro possui uma grande representatividade para o país. Pontua-se assim, que construir indicadores para a mensuração das respostas estratégicas às pressões institucionais para a sustentabilidade em empresas internacionalizadas do setor moveleiro torna-se relevante, à medida que o setor tem se preocupado com as questões sustentáveis e possui um grande número de empresas 
internacionalizadas. Em seguida apresentam-se os procedimentos metodológicos utilizados no desenvolvimento do estudo.

\section{MÉTODO DO ESTUDO}

Com o propósito de atender o objetivo do estudo, desenvolveu-se a pesquisa em duas etapas. A primeira etapa consistiu em uma pesquisa exploratória com abordagem qualitativa e foi operacionalizada a partir da realização de entrevistas e pesquisa bibliográfica. A segunda etapa do estudo caracterizou-se como uma pesquisa de natureza descritiva e abordagem quantitativa sendo concretizada por meio do método survey.

O modelo conceitual da pesquisa baseia-se nas respostas estratégicas às pressões institucionais de Oliver (1991) que originalmente está composto pelas variáveis aquiescência, compromisso, fuga, desafio e manipulação. A sustentabilidade é analisada sob a perspectiva do Triple Botton Line, compreendida pelas dimensões ambiental, social e econômica. A partir dos resultados da etapa qualitativa, da literatura e de dados secundários, desenvolveu-se um instrumento de pesquisa para mensurar quantitativamente as respostas estratégicas às pressões institucionais para a sustentabilidade. As seções a seguir irão detalhar o percurso metodológico adotado.

\subsection{Etapa qualitativa do estudo}

Esta etapa da pesquisa caracterizou-se como de natureza exploratória e investigou o problema sob uma perspectiva qualitativa (Malhotra, 2006). Assim, objetivou-se um maior contato com a realidade das empresas estudadas e a construção do instrumento para coleta de dados na etapa quantitativa. Para tanto, além dos dados primários coletados por meio de entrevistas com três empresas industriais do setor moveleiro. foram realizadas pesquisa bibliográfica e coleta de dados secundários por meio de documentos disponibilizados pelas empresas, como jornais, revistas, informativos, além da consulta nos websites das empresas estudadas.

No que diz respeito a coleta de dados primários, participaram três empresas industriais do setor moveleiro associadas à Associação das Indústrias de Móveis do Estado do Rio Grande do Sul (MOVERGS). Essas foram selecionadas por critério de conveniência e acessibilidade. Foram realizadas entrevistas exploratórias previamente agendadas com gerentes e/ou diretores das organizações. Os dados foram coletados in loco durante o mês de 
setembro de 2014. As entrevistas foram gravadas e posteriormente transcritas e tiveram uma duração média de 45 minutos cada uma.

Para a análise e interpretação dos dados da etapa qualitativa, adotou-se como referência a técnica de análise de conteúdo de Bardin (2010). A operacionalização da análise das entrevistas contou com o auxílio do Software NVIVO 8.0, que "tem a função tanto de validar como de gerar confiança, qualificando o material coletado" (Mozzato \& Grzybovski, 2011, p. 743). Cabe salientar, mais uma vez, que os resultados desta etapa foram utilizados para desenvolver o instrumento de pesquisa para avaliar quantitativamente as respostas estratégicas dadas às pressões institucionais para sustentabilidade.

\subsection{Etapa quantitativa do estudo}

A segunda etapa do estudo caracteriza-se como uma pesquisa de natureza descritiva com abordagem quantitativa (Hair, Babin, Money, \& Samouel., 2005). A concretização da etapa quantitativa da pesquisa utilizou-se do método survey, estruturado a partir de questões com uso de escalas do tipo Likert e razão. O universo da pesquisa foi composto pelas empresas pertencentes à Associação das Indústrias de Móveis do Estado do Rio Grande do Sul (MOVERGS). Para a composição da população-alvo da pesquisa, e posterior, delimitação da amostra, foram selecionadas somente as indústrias de móveis que estavam realizando atividades internacionais, há pelo menos cinco anos. Infere-se que não foram incluídas empresas realizam apenas importação. Neste sentido, mapeou-se um total de 215 empresas associadas à MOVERGS. Destas, apenas 98 empresas mantinham atividades internacionais regulares constituindo assim, a população-alvo do estudo.

Todas as empresas pertencentes à população-alvo foram contatadas por telefone e após procedeu-se com a aplicação do questionário aos respondentes via e-mail com o hiperlink para acesso eletrônico ao questionário que foi administrado por meio da ferramenta Google Drive, juntamente com uma carta convite esclarecendo os objetivos do estudo. Os dados da etapa quantitativa do estudo foram coletados no período de outubro a dezembro de 2014 . Tendo em vista que, das 98 empresas contatadas, 58 delas retornaram o questionário respondido, obteve-se um índice de retorno de 59,18\%, o que pode ser considerado positivo para o estudo. Os dados coletados foram tabulados por meio do programa Microsoft Excel e analisados com o auxílio do software Statistical Package for the Social Sciences - SPSS (versão 21). A técnica de análise fatorial exploratória (AFE) foi utilizada, tendo em vista que 
esta técnica gera novos fatores e tem por objetivo reduzir o número de indicadores de cada variável, ou seja, determinar os construtos fundamentais ou dimensões assumidas inerentes às variáveis originais.

Seguindo as recomendações de Hair, Black, Babin, Anderson, e Tatham (2009), antes de rodar a AFE foram realizados testes estatísticos para verificar a adequação da utilização da análise fatorial para o estudo. Para tanto, os testes indicaram a sua utilização, e a AFE foi realizada de acordo com os seguintes parâmetros: a) Para a extração dos fatores utilizou-se a técnica de análise dos componentes principais; b) Para a rotação dos fatores, foi utilizado o critério Varimax; c) Para a seleção dos fatores utilizou-se o critério Kaiser, com autovalores cuja variância explicada é superior a 1 e retiraram-se as cargas cruzadas maiores que 0,3; d) A qualidade da correlação entre as variáveis foi identificada pelo Kaiser-Meyer-Olkin (KMO) e o teste de esfericidade de Bartlett. Por fim, verificou-se a consistência interna do construto por meio da análise do Alpha de Cronbach.

\subsubsection{Caracterização da amostra}

As 58 empresas pesquisadas localizam-se em 18 municípios do estado do Rio Grande do Sul, sendo que a maioria está localizada nos municípios de Bento Gonçalves $(24,1 \%)$, Lagoa Vermelha (12,1\%), Garibaldi (10,3\%), Flores da Cunha (8,6\%), Farroupilha $(8,6 \%)$ e Antônio Prado (6,9\%), representando 70,6\% da amostra. A predominância da localização das empresas nestes municípios revela o potencial da região e a importância do polo moveleiro do Rio Grande do Sul, o qual ocupa o $2^{\circ}$ lugar no Brasil. Verificou-se que, em média, as empresas estão há 28 anos no mercado, variando entre 7 e 68 anos. Entre estas empresas, verifica-se que muitas atingem o patamar mínimo de experiência internacional estipulado pela pesquisa (5 anos), enquanto outras atingiram o patamar de 30 anos de atuação no mercado externo (em média 12 anos).

A principal linha de produtos das empresas analisadas é a de móveis para dormitórios $(25,9 \%)$, seguida de móveis para cozinhas $(20,7 \%)$. No que tange ao porte das empresas, verificado por meio do número de funcionários, verificou-se que 79,3\% das empresas pesquisadas são de pequeno e médio porte, formado especialmente por empresas de pequeno porte (50\% da amostra). Embora as grandes empresas não apresentem um percentual de destaque, desempenham um importante papel no setor moveleiro no que tange ao atendimento de pressões institucionais, servindo, muitas vezes, de modelo às demais concorrentes. 
A próxima seção, destinada a apresentação dos resultados, expõe a proposição dos indicadores para mensuração das respostas estratégicas para a sustentabilidade em empresas moveleiras internacionalizadas.

\section{APRESENTAÇÃO E DISCUSSÃO DOS RESULTADOS}

Os resultados do estudo se encontram apresentados em três seções. A primeira seção apresenta a construção do instrumento de pesquisa e os indicadores para mensurar as respostas estratégicas às pressões institucionais para a sustentabilidade no setor moveleiro. A segunda discorre sobre a preparação da matriz de entrada de dados. Na terceira seção são demonstrados os indicadores que se ajustaram ao modelo, tendo em vista a aplicação do instrumento junto a 58 empresas internacionalizadas do setor moveleiro do Rio Grande do Sul.

\subsection{Instrumento de pesquisa proposto}

Esta seção tem o propósito de apresentar as variáveis que compõem o instrumento de pesquisa quantitativo desenvolvido com base em Oliver (1991), na literatura e nas entrevistas exploratórias com três empresas do setor moveleiro do RS.

O instrumento de pesquisa desenvolvido para a coleta de dados foi elaborado com base no modelo conceitual de pesquisa e nos resultados encontrados na etapa qualitativa do estudo. Destaca-se que o questionário foi estruturado a partir de perguntas abertas e fechadas, sendo que para compor o bloco relativo às respostas estratégicas às pressões institucionais para sustentabilidade baseou-se na literatura sobre o tema, no modelo de Oliver (1991) e nas entrevistas exploratórias realizadas na fase qualitativa do estudo.

Os resultados da pesquisa qualitativa permitiram concluir que as empresas analisadas apresentam uma vasta experiência nas atividades do setor moveleiro. As três empresas estudadas estão localizadas na Serra Gaúcha, sendo que cada uma delas prioriza a produção de uma linha específica de produtos. Em relação ao número de empregados, elas podem ser classificadas como de médio e grande porte e possuem uma experiência mínima de dez anos em relação às atividades internacionais.

No que tange às práticas sustentáveis, as evidências encontradas demonstram que as empresas têm se preocupado com as questões ambientais, econômicas e sociais no local onde estão inseridas. A visão compartilhada pelas empresas analisadas, a respeito da 
sustentabilidade está alinhada aos preceitos de Elkington (1998). Elas também entendem ser um processo que precisa permear toda a organização. Entre as principais práticas sustentáveis realizadas pelas empresas, destacam-se a preocupação com o ambiente no qual a empresa está instalada, certificações, legislações, inovação dos produtos, tratamento adequado aos resíduos, ações e programas sociais, além da importância atribuída ao um bom relacionamento com os parceiros (clientes, fornecedores) nacionais e internacionais.

Desse modo, pode-se notar que as pressões institucionais para a adoção das práticas sustentáveis estão relacionadas à exigência dos clientes e da sociedade para uma atuação de forma que preserve os recursos naturais e apresente-se de maneira responsável frente às demandas sociais. Por outro lado, as empresas sentem-se pressionadas para atender as exigências dos órgãos públicos e governo para a atuação dentro dos critérios da legislação e adoção de padrões de certificação no seu processo produtivo. O mercado, por sua vez, tem demandado produtos inovadores, com design diferenciado e proveniente de fontes sustentáveis.

A partir das evidências encontradas na literatura e os resultados obtidos na etapa qualitativa construíram-se as 25 variáveis observáveis descritas na Tabela 2. As variáveis estão contidas em 5 variáveis latentes (de acordo com o indicado por Oliver, 1991), propostas para medir o construto relacionado às respostas estratégicas às pressões institucionais para a sustentabilidade.

Destaca-se que a validação após a construção do instrumento de coleta de dados contou com a participação de especialistas das áreas de sustentabilidade, internacionalização e estatística. O grupo de especialistas foi formado por cinco professores doutores com experiência na área do estudo (04), e na área estatística (01), conforme recomendam Hair et al. (2005). Este procedimento de validação buscou verificar a adequação do instrumento de coleta de dados no que se refere ao conteúdo da pergunta, formulação, sequência, formato e layout, dificuldade da pergunta e às escalas utilizadas.

Com base nas sugestões dos especialistas, o questionário foi aprimorado e, em seguida, foi realizado o pré-teste para verificar a sua adequação com os gerentes e/ou diretores de três empresas do setor moveleiro do RS. Após a realização dos ajustes sugeridos pelas empresas na etapa de pré-teste, o questionário foi concluído e procedeu-se ao início da coleta de dados. Pode-se, então, avançar em direção à realização dos procedimentos estatísticos para tratamento dos dados coletados, que se inicia pela preparação da matriz de entrada dos dados 
e em seguida apresenta os resultados da análise fatorial exploratória, detalhadas nas próximas seções.

\section{Tabela 2}

Variáveis referentes às respostas estratégicas às pressões institucionais para a sustentabilidade

\begin{tabular}{|c|c|c|}
\hline $\begin{array}{l}\text { Variáveis } \\
\text { latentes }\end{array}$ & Fator & Variáveis observáveis \\
\hline \multirow{5}{*}{ Aquiescência } & A1 & $\begin{array}{l}\text { A empresa utiliza-se da divulgação de relatórios de sustentabilidade para melhorar a sua } \\
\text { imagem perante a sociedade. }\end{array}$ \\
\hline & A2 & A empresa implantou a ISO 26000 (responsabilidade social) imitando concorrentes. \\
\hline & A3 & $\begin{array}{l}\text { A empresa tem realizado inovação no design de móveis para atender normas } \\
\text { internacionais. }\end{array}$ \\
\hline & A4 & A empresa possui um relacionamento saudável com a comunidade na qual está inserida. \\
\hline & A5 & $\begin{array}{l}\text { A empresa adota o sistema de certificação do conselho de manejo florestal (FSC) para } \\
\text { obter um melhor relacionamento com os clientes. }\end{array}$ \\
\hline \multirow{5}{*}{ Compromisso } & $\mathrm{C} 1$ & $\begin{array}{l}\text { A empresa desenvolveu um programa de cargos e salários para atender as reivindicações } \\
\text { dos funcionários e do sindicato da classe. }\end{array}$ \\
\hline & $\mathrm{C} 2$ & $\begin{array}{l}\text { A empresa tem se preocupado com o tratamento dos resíduos sólidos após exigência dos } \\
\text { órgãos ambientais. }\end{array}$ \\
\hline & $\mathrm{C} 3$ & $\begin{array}{l}\text { A empresa tem apoiado a associação do setor na negociação com agentes de governo para } \\
\text { obter concessões/alterações na cobrança de tributos e taxas sobre a exportação de móveis. }\end{array}$ \\
\hline & $\mathrm{C} 4$ & $\begin{array}{l}\text { A empresa aderiu à norma ISO } 9000 \text { (gestão da qualidade) após detectar problemas na } \\
\text { qualidade de seus produtos. }\end{array}$ \\
\hline & C5 & $\begin{array}{l}\text { A empresa tem se preocupado com a comunidade e seu entorno após a instalação da } \\
\text { fábrica. }\end{array}$ \\
\hline \multirow{5}{*}{ Fuga } & $\mathrm{F} 1$ & $\begin{array}{l}\text { A empresa aderiu ao sistema de gestão de saúde e de segurança ocupacional (OHSAS } \\
18001 \text { - riscos / acidentes), mas nem sempre possui todos os equipamentos de proteção } \\
\text { individual (EPI) em estoque. }\end{array}$ \\
\hline & $\mathrm{F} 2$ & $\begin{array}{l}\text { A empresa desativou o programa de visitas à fábrica em virtude das altas emissões de } \\
\text { ruídos dos equipamentos. }\end{array}$ \\
\hline & $\mathrm{F} 3$ & $\begin{array}{l}\text { A empresa tem recusado pedidos de clientes no exterior em virtude dos aspectos legais } \\
\text { deste novo mercado de atuação. }\end{array}$ \\
\hline & $\mathrm{F} 4$ & $\begin{array}{l}\text { A empresa tem deixado de produzir determinada linha de produtos para exportação em } \\
\text { função das exigências na padronização da embalagem. }\end{array}$ \\
\hline & F5 & $\begin{array}{l}\text { A empresa passou a reutilizar e a reciclar materiais devido às cobranças da sociedade para } \\
\text { o uso adequado dos recursos. }\end{array}$ \\
\hline \multirow{5}{*}{ Desafio } & D1 & $\begin{array}{l}\text { A empresa tem se omitido frente à solicitação da associação do setor para proporcionar } \\
\text { capacitações e treinamentos aos funcionários. }\end{array}$ \\
\hline & D2 & A empresa contestou algumas das legislações instituídas pelo governo. \\
\hline & D3 & $\begin{array}{l}\text { A empresa posicionou-se de forma contrária e adotou providências a um novo acordo } \\
\text { comercial que prejudicaria o setor moveleiro. }\end{array}$ \\
\hline & D4 & A empresa tem postergado a adesão à certificação da ISO 14000 (gestão ambiental). \\
\hline & D5 & A empresa tem contestado reclamações de clientes em relação aos seus produtos. \\
\hline
\end{tabular}




\begin{tabular}{|c|c|l|}
\hline & M1 & $\begin{array}{l}\text { A empresa tem se aliado aos seus concorrentes para negociar a compra da matéria prima } \\
\text { com fornecedores. }\end{array}$ \\
\cline { 2 - 3 } Manipulação & M2 & $\begin{array}{l}\text { A empresa tem atribuído a responsabilidade pela saúde de seus funcionários à gestão } \\
\text { pública. }\end{array}$ \\
\cline { 2 - 3 } & M3 & $\begin{array}{l}\text { A empresa tem se unido aos concorrentes contra a pressão do setor para modernizar o } \\
\text { parque fabril por meio da compra de equipamentos de alta tecnologia. }\end{array}$ \\
\cline { 2 - 3 } & M4 & $\begin{array}{l}\text { A empresa tem desenvolvido campanhas publicitárias de educação e conscientização } \\
\text { ambiental para ser reconhecida pelos consumidores como uma empresa ambientalmente } \\
\text { correta. }\end{array}$ \\
\cline { 2 - 3 } & M5 & $\begin{array}{l}\text { A empresa tem estabelecido parcerias internacionais a fim de atender às exigências legais } \\
\text { e ambientais referentes à internacionalização de suas atividades. }\end{array}$ \\
\hline
\end{tabular}

\subsection{Preparação da matriz de entrada de dados}

Com o propósito de orientar a realização da análise estatística este bloco versa sobre a preparação da matriz de entrada de dados, que segundo Hair et al. (2009) norteia o pesquisador ao atendimento das suposições estatísticas e a identificação de casos atípicos que podem influenciar os resultados de forma desproporcional. Assim, os dados coletados junto à amostra passaram pelos seguintes tratamentos: exame de missing values, identificação de outliers e a normalidade da distribuição dos dados.

Hair et al. (2009) elucidam que o primeiro passo para o exame de dados faltantes é a determinação do tipo de dados envolvidos na pesquisa. Nesta perspectiva, as respostas correspondentes à NA (não se aplica) foram tratadas da mesma forma que os missing values, ou seja, foram substituídos pela média (Hair et al., 2009). Os missing values foram identificados por uma distribuição de frequência simples dos dados faltantes. As observações atípicas ou outliers foram identificados por meio do cálculo Z Score (Escore Z), sendo que todos os dados demonstraram valores menores que 3 (Hair et al., 2005). Com base neste teste não foram encontrados outliers para a amostra.

A normalidade, por sua vez, é vista como uma suposição essencial e caracteriza-se pelo grau em que a distribuição dos dados da amostra corresponde a uma distribuição normal (Hair et al., 2009). Para verificar a normalidade dos dados da amostra foi realizado o teste estatístico Kolmogorov-Smirnov, também conhecido como K-S, cujo resultado demostrou que os dados são normais. A partir do tratamento dos dados, pode-se avançar em direção a caracterização da amostra e a análise fatorial exploratória. 


\subsection{Análise fatorial exploratória}

A análise fatorial exploratória teve o objetivo de verificar se as variáveis identificadas na etapa exploratória do estudo possuem a capacidade de explicar o fenômeno que compõe o construto das respostas estratégicas às pressões institucionais para a sustentabilidade. Como sugerem Hair et al. (2009), procedeu-se os testes estatísticos para se verificar a adequação da utilização da análise fatorial. O índice de Kaiser-Meyer-Olkin (KMO=0,660) de adequação da amostra e o teste de esfericidade de Bartlett (significativo a $\mathrm{p}<0,001$ ) indicaram a fatorabilidade dos dados. Após essa verificação inicial, partiu-se para a identificação das comunalidades apresentadas em cada uma das questões dispostas. Rodaram-se as variáveis observáveis por dimensão (Aquiescência, Compromisso, Fuga, Desafio e Manipulação).

A Análise Fatorial Exploratória foi realizada por meio do método de análise de componentes principais, utilizando o critério a priori para decidir quantos fatores reter e empregando o método rotacional ortogonal varimax para a extração dos fatores, cujo objetivo é explicar a máxima variância total em um número mínimo de fatores. Além disso, para a determinação do número de fatores, utilizou-se da análise da variância total explicada, que deve alcançar no mínimo $60 \%$ da variância acumulada e autovalores maiores do que 1 (Malhotra, 2006). Desta forma, o critério Kaiser foi utilizado para a seleção dos fatores com autovalores cuja variância explicada é superior a 1 e retiraram-se as cargas cruzadas maiores que 0,3 .

Foi necessária a retirada de nove variáveis que não apresentaram cargas fatoriais satisfatórias, além de comunalidades menores que 0,6 e que não tinham, portanto, capacidade para explicar a dimensão. Com relação à Aquiescência retiraram-se as variáveis A2 e A4. No que tange ao Compromisso, retirou-se a variável C3. Com relação à Fuga, retirou-se a variável F2. A estratégia Desafio, por sua vez, demandou a retirada das variáveis D1, D4 e D5. No último dos fatores, a Manipulação, retiraram-se as variáveis M1 e M2. Em seguida, rodou-se o construto final ajustado (Aquiescência, Compromisso, Fuga, Desafio e Manipulação), a partir do que se verificou a necessidade da retirada de mais uma variável relacionada à resposta estratégica Compromisso. Assim, procedeu-se a retirada da variável C1.

Para tanto, atendidos os parâmetros da análise fatorial exploratória restaram as variáveis A1, A2 e A3 (Aquiescência), C2, C4 e C5 (Compromisso), F1, F3, F4 e F5 (Fuga), 
D2 e D3 (Desafio) e as variáveis M3, M4 e M5 (Manipulação). Deste modo, a Tabela 3, apresenta o construto final, com os fatores extraídos, os indicadores e suas respectivas cargas fatoriais e o total de variância explicada.

\section{Tabela 3}

Fatores relacionados às respostas estratégicas às pressões institucionais para a sustentabilidade

\begin{tabular}{|c|c|c|c|c|}
\hline Fatores $^{1}$ & Indicadores & $\begin{array}{c}\text { Cargas } \\
\text { fatoriais }\end{array}$ & $\begin{array}{c}\text { Auto } \\
\text { valores }\end{array}$ & $\begin{array}{c}\% \text { da } \\
\text { Variância } \\
\text { explicada }\end{array}$ \\
\hline $\begin{array}{l}\text { Aquies- } \\
\text { cência }\end{array}$ & $\begin{array}{l}\text { A1 - A empresa utiliza-se da divulgação de } \\
\text { relatórios de sustentabilidade para melhorar a sua } \\
\text { imagem perante a sociedade } \\
\text { A3 - A empresa tem realizado inovação no design } \\
\text { de móveis para atender normas internacionais } \\
\text { A5 - A empresa adota o sistema de certificação } \\
\text { do conselho de manejo florestal (FSC) para obter } \\
\text { um melhor relacionamento com os clientes }\end{array}$ & $\begin{array}{l}0,684 \\
0,625\end{array}$ & 3,613 & 18,93 \\
\hline $\begin{array}{c}\text { Compro- } \\
\text { misso }\end{array}$ & $\begin{array}{l}\text { C2 - A empresa tem se preocupado com o } \\
\text { tratamento dos resíduos sólidos após exigência } \\
\text { dos órgãos ambientais } \\
\text { C4 - A empresa aderiu à norma ISO } 9000 \text { (gestão } \\
\text { da qualidade) após detectar problemas na } \\
\text { qualidade de seus produtos } \\
\text { C5 - A empresa tem se preocupado com a } \\
\text { comunidade e seu entorno após a instalação da } \\
\text { fábrica }\end{array}$ & $\begin{array}{l}0,756 \\
0,750\end{array}$ & 1,166 & 11,31 \\
\hline Fuga & $\begin{array}{l}\text { F1 - A empresa aderiu ao sistema de gestão de } \\
\text { saúde e de segurança ocupacional (OHSAS } 18001 \\
\text { - riscos / acidentes), mas nem sempre possui } \\
\text { todos os equipamentos de proteção individual } \\
\text { (EPI) em estoque } \\
\text { F3 - A empresa tem recusado pedidos de clientes } \\
\text { no exterior em virtude dos aspectos legais deste } \\
\text { novo mercado de atuação } \\
\text { F4 - A empresa tem deixado de produzir } \\
\text { determinada linha de produtos para exportação } \\
\text { em função das exigências na padronização da } \\
\text { embalagem } \\
\text { F5 - A empresa passou a reutilizar e a reciclar } \\
\text { materiais devido às cobranças da sociedade para o } \\
\text { uso adequado dos recursos }\end{array}$ & 0,766 & 2,299 & 13,16 \\
\hline
\end{tabular}


(continuação)

\begin{tabular}{|c|c|c|c|c|}
\hline \multirow[b]{2}{*}{ Desafio } & \multicolumn{4}{|c|}{$\begin{array}{l}\text { D2 - A empresa contestou algumas } \\
\text { das legislações instituídas pelo } 0,740\end{array}$} \\
\hline & $\begin{array}{l}\text { D3 - A empresa posicionou-se de } \\
\text { forma contrária e adotou } \\
\text { providências a um novo acordo } \\
\text { comercial que prejudicaria o setor } \\
\text { moveleiro }\end{array}$ & 0,789 & 1,292 & 11,60 \\
\hline Manipulação & $\begin{array}{l}\text { M3 - A empresa tem se unido aos } \\
\text { concorrentes contra a pressão do } \\
\text { setor para modernizar o parque } \\
\text { fabril por meio da compra de } \\
\text { equipamentos de alta tecnologia } \\
\text { M4 - A empresa tem desenvolvido } \\
\text { campanhas publicitárias de } \\
\text { educação e conscientização } \\
\text { ambiental para ser reconhecida } \\
\text { pelos consumidores como uma } \\
\text { empresa ambientalmente correta } \\
\text { M5 - A empresa tem estabelecido } \\
\text { parcerias internacionais a fim de } \\
\text { atender às exigências legais e } \\
\text { ambientais referentes } \\
\text { internacionalização de suas } \\
\text { atividades }\end{array}$ & 0,733 & 1,814 & 12,89 \\
\hline
\end{tabular}

Método de extração ${ }^{1}$ : Análise de componentes principais;

Método de rotação: varimax com normalização Kaiser

$\mathrm{KMO}=0,660$; Teste de Barlett com significância $\mathrm{p}=0,000$

Alpha de Cronbach total 0,7

Os resultados da análise fatorial, dispostos na Tabela 3, indicam que as cinco dimensões (fatores) foram mantidas, no entanto foram eliminadas 10 variáveis por não atenderem aos parâmetros da análise fatorial exploratória, como explicado anteriormente. Desta forma, restaram 15 variáveis que explicam 67,89\% da variância total dos dados.

O primeiro fator, denominado aquiescência, abrange três variáveis que se referem à publicação de relatórios de sustentabilidade, inovação no design de móveis e a adoção de certificação florestal com vistas ao atendimento das demandas sustentáveis. Este fator constitui-se como o de maior representatividade no construto. $\mathrm{O}$ segundo fator, intitulado como compromisso, encontra-se estruturado por três variáveis que possuem o foco no 
tratamento dos resíduos sólidos, adoção da norma ISO 9000 e a preocupação com a comunidade e seu entorno.

A fuga, constitui-se na dimensão que agrega o maior número de variáveis do construto proposto, sendo composto por quatro variáveis ao final. Esta categoria representa a preocupação com a saúde e segurança ocupacional dos trabalhadores, as questões relacionadas aos aspectos legais, tanto na forma da lei como de exigências para internacionalização das atividades. Além disso, preocupa-se também com a necessidade de reutilizar e reciclar materiais, o que contribui para um ambiente sustentável.

$\mathrm{O}$ quarto fator, denominado desafio, compreende a dimensão que reuniu o menor número de variáveis, sendo constituído por apenas dois indicadores do construto. Os indicadores que compõem este fator tratam da posição contrária dos empresários a instituição de acordos comerciais e legislações que poderiam prejudicar o setor moveleiro.

Por fim, o quinto fator, conhecido como manipulação, abrange três variáveis relacionadas às pressões do setor para a modernização do parque fabril, campanhas publicitárias com o intuito de expor aos clientes o que a empresa tem realizado em termos sustentáveis e a busca de parcerias internacionais. Essa dimensão consiste na busca de respostas mais ativas às pressões institucionais sofridas pelo setor e representa $12,89 \%$ do total da variância explicada.

Após a descrição dos cinco fatores que compõem o construto respostas estratégicas às pressões institucionais para a sustentabilidade, verificou-se o grau de consistência, ou seja, a confiabilidade do construto, por meio do cálculo do Alpha de Cronbach. Obteve-se o alpha igual a 0,731, o que indica a consistência interna do modelo, nos padrões de Hair et al. (2009).

Cabe salientar que o modelo originalmente proposto por Oliver (1991), não traz os indicadores que mensuram as respostas estratégicas às pressões institucionais para a sustentabilidade, apenas as cinco dimensões (aquiescência, compromisso, fuga, desafio e manipulação) que foram mantidas neste estudo. Assim, uma das contribuições do estudo foi propor indicadores que permitam mensurar as respostas estratégicas no que tange à sustentabilidade. Desta forma, a partir do modelo original, da literatura e das entrevistas exploratórias realizadas na etapa qualitativa foi possível elaborar um instrumento de mensuração que, após a análise fatorial exploratória, foi resumido em 15 indicadores para mensurar os cinco fatores que já tinham sido definidos por Oliver (1991). 
Destaca-se que se optou por manter as mesmas dimensões de respostas estratégicas definidas por Oliver (1991), considerando que estas são aceitas pela literatura (Sutter et al., 2013; Cao \& Pederzoli, 2013), bem como os fatores extraídos demostraram o ajuste dos indicadores nas respectivas dimensões propostas.

\section{CONSIDERAÇÕES FINAIS}

Inseridas em um ambiente dinâmico e em constantes transformações, as organizações contemporâneas precisam manter-se atualizadas, de forma a responderem estrategicamente às pressões institucionais recebidas do governo, clientes, órgãos públicos, sociedade e de seu próprio segmento para a institucionalização de práticas sustentáveis e o alcance de novos mercados na arena global. Desta forma, o presente estudo apresentou como objetivo construir indicadores para a mensuração das respostas estratégicas às pressões institucionais para a sustentabilidade em empresas internacionalizadas do setor moveleiro.

Os resultados obtidos na fase qualitativa foram essenciais para conhecer as empresas em relação à atuação no exterior, as práticas sustentáveis e pressões institucionais, com vistas à elaboração do instrumento de pesquisa para a etapa quantitativa do estudo. Os resultados da análise fatorial exploratória indicaram a manutenção das cinco dimensões (fatores) relacionada às respostas estratégicas, que explicam 67,89\% da variância total dos dados. Além disso, obteve-se um Alpha de Cronbach de 0,731, o que indica consistência interna do modelo.

Assim, a construção de uma escala que mensure as respostas estratégicas às pressões institucionais para a sustentabilidade apresenta contribuições acadêmicas por meio da proposição e aplicação de um instrumento de pesquisa quantitativo. De acordo com a literatura sobre o tema, os estudos que abordam a perspectiva das respostas estratégicas, propostas por Oliver (1991), são em sua maioria estudos qualitativos.

Como contribuição para o meio empresarial, a pesquisa amplia o conhecimento sobre as práticas sustentáveis e pressão institucional as quais o setor está exposto. Assim, é esperado que os gestores tenham a possibilidade de usar tal conhecimento de forma a qualificar as respostas que vêm sendo dadas às pressões institucionais. Aqui, infere-se que conhecimento pode gerar reflexão sobre práticas e, quiçá, a adoção de novas e melhores posturas gerenciais. 
Sugere-se que estudos futuros, realizem entrevistas exploratórias com um número maior de empresas moveleiras, a fim de aprofundar os resultados e assim poder propor - se necessário - outros indicadores para mensurar as respostas estratégicas às pressões institucionais para a sustentabilidade nas empresas investigadas.

Recomenda-se também a ampliação do estudo para outros contextos do setor moveleiro brasileiro, pois se entende que as particularidades da região na qual as empresas estão instaladas podem revelar diferentes posições de respostas estratégicas. Além disso, sugere-se realizar a pesquisa em outros setores da economia, o que pode trazer novos comportamentos frente às pressões institucionais para a adoção de práticas sustentáveis.

Por fim, sugere-se a realização da pesquisa em outros países a fim de traçar um paralelo entre o comportamento das respostas estratégicas para a sustentabilidade no que tange às pressões institucionais em empresas internacionalizadas entre países emergentes e desenvolvidos e, também, diante dos diferentes modos de entrada para atuação no mercado internacional.

\section{REFERÊNCIAS}

Ahmed, F. U., \& Brennan, L. (2019). An institution-based view of firms' early internationalization Effectiveness of national export promotion policies. International Marketing Review.

$\begin{array}{lllll}\text { Aprendendo A } & \text { Exportar. } & \text { Móveis. } & \text { (2014). Disponível }\end{array}$ <http://www.aprendendoaexportar.gov.br/moveis/>.

Arruda, G. L. R. C. (2009). O design na indústria moveleira brasileira e seus aspectos sustentáveis: estudo de caso no polo moveleiro de Arapongas-Pr. 2009. $121 \mathrm{f}$. Dissertação (Mestrado) - Universidade Estadual Paulista Júlio de Mesquita Filho, Bauru.

Associação Brasileira das Indústrias do Mobiliário - ABIMÓVEL. (2014). Dados do setor. Disponível em: <http://www.abimovel.com>.

Associação Das Indústrias De Móveis Do Estado Do Rio Grande Do Sul - MOVERGS. (2014). Dados do setor moveleiro. Disponível em: <http://www.movergs.com.br/numerossetor>.

Banco Nacional de Desenvolvimento Econômico e Social - BNDES. (2005). BNDES setorial 37. In: A competitividade da indústria de móveis do Brasil: situação atual e perspectivas. Rio de Janeiro, 2013. p. 227-272.

Barbieri, J. C., Vasconcelos, I. F. G. de, Andreassi, T. \& Vasconcelos, F. C. de. (2010). Inovação e sustentabilidade: novos modelos e proposições. Revista de Administração de Empresas - RAE, v. 50, n. 2, p. 146-154, abr./jun.

Bardin, L. (2010). Análise de conteúdo. 4. ed. Lisboa: Edições 70.

Cao, L., \& Pederzoli, D. (2013). International retailers' strategic responses to institutional environment of emerging Market. International Journal of Retail \& Distribution Management, v. 41, n. 4 
Carneiro, J., \& Dib, L. A. (2007). Avaliação comparativa do escopo descritivo e explanatório dos principais modelos de internacionalização de empresas. Revista Eletrônica de Negócios Internacionais da ESPM, v. 2, n. 1, p. 1-25, jan./jun.

Carvalho, C. A., Vieira, M. M. F., \& Silva, M. G. (2012). A trajetória conservadora da Teoria Institucional. Revista Eletrônica de Gestão Organizacional, v. 10, n. especial, p. 469- 496, dez.

Carvalho, H. (2010). Neoinstitucionalismo e suas implicações na adoção de práticas socioambientais nas organizações: estudo de caso no segmento de comércio exterior. 2010. 84 f. Dissertação (Mestrado em Administração) - Fundação Instituto Capixaba de Pesquisa em Contabilidade, Economia e Finanças, Vitória.

Ciasullo, M. V., Montera, R., Cucari, N., \& Polese, F. (2020). How an international ambidexterity strategy can address the paradox perspective on corporate sustainability: Evidence from Chinese emerging market multinationals. Business Strategy and the Environment.

Cunha, C. F., Spers, E. E., \& Zylbersziajn, D. (2011). Percepção sobre atributos de sustentabilidade em um varejo supermercadista. Revista de Administração de Empresas, v. 51, n. 6, p. 542-552.

Deng, P., \& Zhang, S. (2018). Institutional quality and internationalization of emerging market firms: Focusing on Chinese SMEs. Journal of Business Research, v.92, p.279-289.

DiMaggio, P. J., \& Powell, W. W. (2005). A gaiola de ferro revisitada: isomorfismo institucional e racionalidade coletiva nos campos organizacionais. Revista de Administração de Empresas RAE, São Paulo, v. 45, n. 2, p. 74-89, abr./jun.

Elkington, J. (1998). Cannibals with forks: the triple bottom line of 21st century business. Canadá: NSP.

Fleury, A. Fleury, M. T. L. (2012). Globalização e internacionalização: a perspectiva dos países emergentes. (p. 25-50). In: FLEURY, M. T. L. Multinacionais brasileiras: competências para a internacionalização. Rio de Janeiro: Editora FGV.

Floriani, D. E. (2010). O grau de internacionalização, as competências e o desempenho da PME Brasileira. 2010. 311 f. Tese (Doutorado em Administração) - Universidade de São Paulo, São Paulo.

Hair, J. F., Black, W. C., Babin, B. J., Anderson, R. E. \& Tatham, L. R. (2009). Análise multivariada de dados. 6. ed. São Paulo: Bookman.

Hair, Jr. J., F., Babin, B., Money, A. H., \& Samouel, P. (2005). Fundamentos de métodos de pesquisa em administração. Porto Alegre: Bookman, 2005.

Leão, M. S., \& Naveiro, R. M. (2014). Móveis: indústria de móveis mostra competitividade da madeira brasileira, Painel Florestal 2010. Disponível em: 〈http//www.remade.com.br>.

Luo, Y.; Zhang, H. (2016). Emerging Market MNEs: Qualitative Review and Theoretical Directions. Journal of International Management, v.22, p.333-350, 2016.

Machado-da-Silva, C. L., \& Fonseca, V. S. (2010). Competitividade organizacional: uma tentativa de reconstrução analítica. Revista de Administração Contemporânea, Curitiba, ed. especial, art. 2, p. 33-49.

Machado-da-Silva, C. L., Fonseca, V. S. da, \& Crubellate, J. M. (2010). Estrutura, agência e interpretação: elementos para uma abordagem recursiva do processo de institucionalização. Revista de Administração Contemporânea, Curitiba, ed. especial, art. 4, p. 77-107.

Maffessoni, D. (2012). Análise da situação ambiental das indústrias do pólo moveleiro de Bento Gonçalves. 2012. 102 f. Dissertação (Mestrado em Engenharia) - Universidade Federal do Rio Grande do Sul, Porto Alegre. 
Malhotra, N. K. (2006). Pesquisa de marketing: uma orientação aplicada. 4. ed. Porto Alegre: Bookman.

Meyer, J. W., \& Rowan, B. (1977). Institutionalized organizations: formal structure as myth and ceremony. American Sociological Review, v. 83, n. 2, p. 340-363.

Mozzato, A. R, \& Grzybovski, D. (2011). Análise de conteúdo como técnica de análise de dados qualitativos no campo da administração: potencial e desafios. Revista de Administração Contemporânea, v.15, n. 4, p. 731-747, Jul./Ago.

Oliver, C. (1991). Strategic responses to institucional processes. Academy of Management Review, v. 16, n. 1, p. 145-179.

Santos, M.S. (2013). Instituições e estratégia como prática: uma análise das estratégias de aquisição de matéria-prima dos produtores de biodiesel da região sul do Brasil. 2013. 263 f. Tese (Doutorado em Administração) - Universidade Federal do Rio Grande do Sul, Porto Alegre.

Scherer, F. L. (2007). Negócios internacionais: a consolidação de empresas brasileiras de construção pesada em mercados externos. 2007. 329 f. Tese (Doutorado em Administração) - Universidade Federal de Minas Gerais, Belo Horizonte.

Scott, W. R. (2008). Institutions and organizations: ideas and interests. London: Sage publications.

Scott, W. R., Ruef, M., Mendel, P. J., \& Caronna, C. A. (2001). Institutional Change and Healthcare Organizations: From Professional Dominance to Managed Care. Chicago, University of Chicago Press.

Selznick, P. A. (1972). Liderança na administração: uma interpretação sociológica. Rio de Janeiro: FGV.

Sutter, C. J., Webb, J. W., Kistruck, G. M. \& Bailey, A.V.G. (2013). Entrepreneurs' responses to semi-formal illegitimate institutional arrangements. Journal of Business Venturing, v. 28, p. 743-758.

Vidal, D. F., Barbosa, J. G. P., \& Bouzada M. A. C. A. (2013). Relação entre pressão ambiental e comportamento estratégico: uma pesquisa em MPE do setor de autopeças. In: Encontro de Estudos em Estratégia, 6., 2013, Bento Gonçalves. Anais... Bento Gonçalves: ANPAD. 